\title{
Risk Control Management of New Rural Cooperative Financial Organizations Based on Mobile Edge Computing
}

\author{
Fanrong Kong ${ }^{1}$ and Hongxia Lu (iD) ${ }^{2}$ \\ ${ }^{1}$ School of Wealth Management, Nanchang Institute of Science and Technology, Nanchang 330108, Jiangxi, China \\ ${ }^{2}$ School of Management, Wuhan Donghu University, Wuhan 430212, Hubei, China \\ Correspondence should be addressed to Hongxia Lu; luhx@wdu.edu.cn
}

Received 21 June 2021; Accepted 6 August 2021; Published 24 September 2021

Academic Editor: Sang-Bing Tsai

Copyright (C 2021 Fanrong Kong and Hongxia Lu. This is an open access article distributed under the Creative Commons Attribution License, which permits unrestricted use, distribution, and reproduction in any medium, provided the original work is properly cited.

\begin{abstract}
Rural cooperative financial organization is a new type of cooperative financial organization in recent years. It is a community financial institution created by farmers and small rural enterprises to voluntarily invest in shares in order to meet the growing demand for rural financing. However, this financial organization has many flaws in the design of the system; it has not promoted the better development of rural mutual fund assistance. In addition, mobile edge computing (MEC) can be used as an effective supplement to mobile cloud computing and has been proposed. However, most of the current literature studies on cloud computing provide computing offload just to propose a network architecture, without modeling and solving to achieve. In this context, this paper focuses on the practical application of MEC in the risk control of new rural cooperative financial organizations. This paper proposes a collaborative LECC mechanism based on machine learning under the MEC architecture. The experimental simulation shows that the HR under the LECC mechanism is about $17 \%-23 \%, 46 \%-69 \%$, and $93 \%-177 \%$ higher than that of LENC, LRU, and RR, respectively. It is unrealistic to want to rely on meager loan interest for long-term development. The most practical way is to increase the income level of the organization itself.
\end{abstract}

\section{Introduction}

Effective improvement of the rural financial system is a basic condition for promoting agricultural development. As one of the most important resources in a market economy, it directly affects the economic growth rate and quality of an economy. In theory, my country's rural financial organization system is perfect, but there are still many problems and shortcomings in the actual operation. In addition, new rural cooperative financial organizations such as poverty alleviation funds and cooperatives have also become active.

In the context of large-scale mobile device access and rapid growth of communication capacity requirements, small cell will realize ultraintensive deployment in the nextgeneration mobile communication system (5G). The storage and computing resources of the small cell can provide ubiquitous computing support [1]. This solution will not only increase the load but also bring greater energy consumption problems [2]. Many researchers have proposed mobile edge caching and computing solutions to solve the above problems [3, 4]. However, existing mobile edge caching and computing solutions also exist. Edge caching solutions are mostly based on fixed network topologies, ignoring user mobility to solve problems in 5G networks [5]. The use of renewable energy for power supply is a feasible solution. However, the randomness of the arrival of renewable energy has led to the dynamic nature of the computing capabilities of edge cloud servers, making it difficult to apply existing off-grid-based computing offloading strategies $[6,7]$.

In Shen et al.'s research, they analyzed the problem that insurance companies allocate their wealth in risk-free bonds and risk assets under random factors. The drift and fluctuation of these assets depend on a factor process. The classical solution of HJB PDE is discussed, and the verification theorem is proved [8]. In order to make the cost of 
software risk control and software risk exposure, a multiobjective risk control model of the software project is proposed. Based on this, Ge et al. proposed another multiobjective risk control model [9]. Ramlatchan et al. systematically reviewed and evaluated the safety of Sophora flavescens from literature sources, modern literature, toxicological literature, and toxicological research. Finally, it is recommended to strictly follow the recommended pharmacopoeia under the guidance of a physician. Patients should not believe in folk remedies and prescriptions and use them at will to prevent cold and treat laryngitis. In addition, in further research, research on traditional treatment methods should be strengthened, and research on the toxic substance basis and mechanism of action of toxic amygdalin should be strengthened $[10,11]$.

A standardized internal governance structure is the core of its sustainable development. The practical application of MEC in the risk control of new-type rural cooperative financial organizations and a good operating mechanism are one of the basic driving forces for the development of rural mutual fund organizations. A good operating mechanism is one of the basic driving forces for the development of rural mutual fund organizations. On this basis, the role of rural mutual capital organizations in serving farmers can be brought into full play. Aiming at the bottleneck of the backhaul link capacity and huge energy consumption in the $5 \mathrm{G}$ network, this paper studies from the perspective of MEC and proposes a LECC mechanism.

\section{Proposed Method}

2.1. MEC Technology. MEC represents the key technology and architecture concepts to realize the evolution to $5 \mathrm{G}$ $[12,13]$. Because it helps to promote the transition of mobile networks to the software programmable world and helps to meet $5 \mathrm{G}$ in terms of throughput, network latency, scalability, and automation [14], MEC has been identified as one of the key technologies of the $5 \mathrm{G}$ network by the European 5G PPP research institute $[15,16]$. The infrastructure carrying MEC and NFV is very similar. Therefore, by hosting virtual network functions and MEC applications on the same platform $[17,18]$, operators can maximize the reuse of NFV infrastructure and facility management and get the maximum investment from the benefit. The efficient and seamless integration on the edge computing platform enables them to play a complementary and profitable role in their respective business models and better monetize the mobile broadband experience. The development of MEC has brought new value chains, new business opportunities, and numerous new application cases to many industries $[19,20]$.

2.2. MEC Architecture. The second part is the MEC application platform. This part is responsible for hosting applications. The details of the MEC infrastructure include the connection with the wireless network element or network and the interface with the host infrastructure management system. There is no specification given by ETSI. Virtualization manager provides flexible and efficient multitenant, runtime, and hosting environment for applications by providing infrastructure services. IAA controllers provide available resources and security for applications and platforms. In the future version of the MEC standard, platform service facilities allow application developers to control processing power, storage, and operating system at different levels.

This deployment scheme enables local related fast services to be provided directly from the base station cluster. The widespread deployment of MEC in the future will accelerate the development of mobile communications and internet services. The MEC cooperative caching mechanism proposed in this paper is called LECC (learning-based cooperative caching) mechanism. The application scenarios of MEC in future mobile communication systems mainly include the following aspects: dynamic content optimization: traditional content optimization solutions mainly use users stored in a database.

The output of a set of edge nodes in related services is determined by a feasible solution with the following constraints.

Feasibility of communication resources: for business, the total number of communication resource allocations of all relevant numbers of available communication resources is

$$
\sum_{\forall n \in N} \sum_{\forall m \in N} I_{m}^{n} \times P_{m}^{n} \leq P
$$

Feasibility of computing resources:

$$
\sum_{\forall n \in N} I_{m}^{n} \times Q_{m}^{n} \leq Q_{M}, \quad \forall M .
$$

Processing data guarantee:

$$
\sum_{\forall n \in M} I_{m}^{n} \times D_{m}^{n} \leq D^{n}, \quad \forall N .
$$

\subsection{New Rural Cooperative Financial Organization.} According to 2014 Central Document No. 1, the new rural cooperative financial organizations must meet the following four requirements: one is membership and closeness. The membership system refers to whether members are limited to holding shares or borrowing money so that capital cooperation is controlled within the scope of acquaintances. Mutual recognition of personnel can greatly reduce financial risks and reflect the limited and fixed nature of personnel. Closeness refers to geographical closeness, that is, the development of members in a certain area, with townships or administrative villages as the unit. Farmers must invest in obtaining membership qualifications when joining new rural cooperative financial organizations and must not recruit nonmembers to join the society. Provide financial services to members. Second, we will not draw loans from external storage, that is, a mutual fundraising activity carried out within a fixed membership and within the region to ensure the safety of funds and not to save and lend to nonmembers. If you break through this boundary at will, it is likely to become a random fundraising. Third, do not pay a fixed return. The new rural cooperative financial organization follows the principle of the cooperative system, sharing benefits and sharing risks, and does not give fixed returns to the 
members' funds it absorbs, and it pays no dividends. Fourth, democratic management and standardized operation: adhere to voluntary membership, freedom of retirement, equal status of members, and surplus distribution in accordance with rules and regulations. The "new type" of the new rural cooperative financial organization is mainly reflected in the following: first, it is different from the traditional rural credit cooperatives and rural cooperative foundations. With the economic development and the continuously adjusted national financial policy, the rural credit cooperative system has changed many times and has already changed. As a commercial financial institution, it is far from being a farmer credit cooperative organization. However, due to excessive government intervention, the rural cooperation foundation caused financial problems and was liquidated. Therefore, the new-type rural cooperative financial organization is not to build these two types of institutions, and the old way must not be taken for the second time. Second, unlike the rural financial institutions such as small loan companies and village and town banks that have emerged actively in recent years, these agricultural financial institutions are commercial financial institutions. Third, the new-type rural cooperative financial organization is based on the supply and marketing cooperatives and farmers' professional cooperatives and is established by relying on them. As the main body of the financial industry, its ownership is in the hands of community farmers. This is a new type of rural cooperative financial organization that should be distinguished from commercial financial institutions. Therefore, the new rural cooperative financial organization is also a new type of rural collective economic organization. Table 1 shows the comparison between the new rural cooperative financial organizations and commercial financial institutions.

To sum up, the connotation of the new rural cooperative financial organization is adhere to the membership system and closeness, and under the premise of not absorbing external storage and lending and not paying a fixed return, the rural disadvantaged groups will voluntarily form a joint organization to solve their own credit difficulties, a cooperative economic organization with mutual funding assistance and democratic management. Therefore, the rural mutual funds, poverty alleviation, and mutual aid funds mentioned in this article and the credit cooperation relying on the development of professional farmers' cooperatives and supply and marketing cooperatives are all new rural cooperative financial organizations.

\section{Experiments}

3.1. Experimental Data. The research scope is mainly in several provinces of China, and the sample is representative. This time, 400 questionnaires were issued, 320 were returned, 320 valid questionnaires, and the effective response rate was $88.67 \%$. Basic statistics of the sample: the basic information of the surveyed samples is shown in Table 2.

3.2. Experimental Environment. The practical work of the new rural cooperative financial organizations has been carried out in various parts of the country, but at present, there is still no special institution to statistically organize relevant data. Based on the opinions of the Anhui Provincial Agriculture Committee and the Provincial Supply and Marketing Cooperatives, this article selects to conduct indepth investigations on Tianchang City and Fengyang County of Yingzhou city, Yingdong District of Fuyang city, and Qianshan County and Taihu County of Anqing city. The project uses a mobile ad hoc network simulation platform to model and analyze the simulation network, which is the evaluation basis for the index detection of the simulation network. On the one hand, the network simulation platform provides corresponding solutions for networks with complex design architectures and topologies. On the other hand, simulation lies between cost and complexity and the accuracy of the results, and a solution with higher simulation accuracy must be found. As a simulation platform for wireless networks and the performance results of each simulation tool are different from each other, determining which simulation platform to use and what accurate results it provides is often difficult and laborious. Therefore, the establishment of a mobile ad hoc network simulation platform is the first condition for analyzing and evaluating network indicators. The use of a suitable mobile ad hoc network simulation platform is one of the focuses of research on large-scale dynamic real-time networking.

Software represented by OPNET, QualNet, and NS3 occupies the mainstream position in the existing network simulation. Due to the existence of the Education Edition, OPNET has attracted a large number of users. Users set up community discussions to make OPNET easier to learn and use in the process of use. Users are more adaptable to OPNET's interface, have deeper functional understanding, and are more proficient in operation. However, QualNet is too expensive in terms of price. Except for large enterprises and research and technical personnel who can use it, the corresponding resources for writing this article are too small. NS3 itself is open source and free, but NS3 has to completely define its own in terms of constructing simulation scenarios and corresponding nodes, so the complexity is greatly increased. Therefore, this article uses the OPNET network simulation platform as a dynamic real-time networking network simulation platform.

\section{Discussion}

4.1. Analysis of Single-Task Dynamic Programming Results. First, consider single business. Because of only one service, the target service $n$ can use all available channel resources from the service edge computing nodes, or it can use all available computing resources of each edge node. Its formula is expressed as

$$
\min \max _{\forall m \in M} I_{m}^{n} \times\left(\frac{D_{m}^{n}}{P_{m}^{n} \times r_{m}^{n}}+\frac{C_{m}^{n}}{Q_{m}^{n} \times q_{m}^{n}}\right) .
$$

By the same token, for this business, all collaborative edge computing nodes are available. Therefore, the algorithm is optimal. Input edge calculation node number 
TABLE 1: The comparison of new rural cooperative financial organizations and commercial financial institutions.

\begin{tabular}{|c|c|c|}
\hline Types & New rural cooperative financial organization & Commercial financial institution \\
\hline Service object & Membership system, providing financial services only to members & Services for both owners and fund users \\
\hline Business objectives & Mutual funding assistance to provide financial services for members & $\begin{array}{r}\text { Adhere to th } \\
\text { ma? }\end{array}$ \\
\hline & Democratic management, one person, one vote & Stock right con \\
\hline & & \\
\hline & & Capital, \\
\hline $\begin{array}{l}\text { Distribution } \\
\text { principle }\end{array}$ & $\begin{array}{c}\text { Dividends are nondividend and are returned based on transaction } \\
\text { volume }\end{array}$ & Dividends by shares, accumulated by shares \\
\hline
\end{tabular}

TABLE 2: The basic characteristic of farmers.

\begin{tabular}{|c|c|c|c|}
\hline Project & Classification & Surveyed farmers (people) & Proportion (\%) \\
\hline \multirow{2}{*}{ Sex } & Male & 178 & 55.6 \\
\hline & Female & 142 & 44.4 \\
\hline \multirow{4}{*}{ Age } & $\leq 30$ & 63 & 19.7 \\
\hline & $31-40$ & 109 & 34.1 \\
\hline & $41-50$ & 95 & 29.7 \\
\hline & $\geq 50$ & 20 & 16.5 \\
\hline \multirow{3}{*}{ Length of residence } & $5-10$ years & 75 & 32.8 \\
\hline & $11-20$ years & 95 & 29.7 \\
\hline & $\geq 20$ years & 120 & 37.5 \\
\hline \multirow{4}{*}{ Marital status } & Married & 280 & 87.5 \\
\hline & Divorced & 8 & 2.5 \\
\hline & Unmarried & 23 & 7.3 \\
\hline & Widowed & 9 & 2.7 \\
\hline \multirow{4}{*}{ Education } & Elementary and below & 34 & 10.6 \\
\hline & Junior high school & 210 & 65.6 \\
\hline & High school & 43 & 13.4 \\
\hline & Bachelor and above & 34 & 10.4 \\
\hline \multirow{4}{*}{ Annual pure input } & $\leq 10,000$ & 76 & 23.8 \\
\hline & $10-20$ thousand & 145 & 45.3 \\
\hline & $21-50$ thousand & 69 & 21.6 \\
\hline & $\geq 50$ thousand & 30 & 9.3 \\
\hline
\end{tabular}

$M=5-100$, calculation data $C=30 \mathrm{MB}$, data rate $\mathrm{RM}$ is set to a $5 * 1$ array of five random numbers from $500 \mathrm{~Hz}$ to $1500 \mathrm{~Hz}$, fixed communication overhead is set to $0.002 \mathrm{MB}$, and the specific output is shown in Figure 1.

Figure 1 shows the task offloading options in different situations, simulating the situation when the task unloading quantity is 30 (the third line in Figure 1), 70 (the second line in Figure 1), and 100 (the first line in Figure 1); the choice of task offloading is also different, but the number of selected offloading nodes has not increased, all are 5, which can be interpreted as the minimum considering the communication delay, and we cannot choose too many small nodes to offload tasks. When the number of nodes is less than or equal to 2 , the optimal delay is still very large. This is mainly because the number of small edge nodes is too small, and there are no more task nodes to choose. If it is greater than 2, the optimization delay is significantly reduced. As the number of small nodes continues to increase, task nodes have more and better selection strategies to optimize delay. This is because it continues to increase and is not selected by other task nodes, so the delay will not continue to be reduced.

4.2. Performance Evaluation. The five cache mechanisms under the change of the MEC cache space are shown.

As shown in Figure 2, the number of service contents ranges from 500 to 5000 , the cost coefficient $\eta$ is fixed at 20, the skew coefficient $\beta$ is 0.55 , and the cache space of each MEC server is fixed at 50 .

\subsection{Analysis of Survey Data on the Operation of Financial} Organizations. Farmers borrow frequently. Table 3 shows the number of borrowings by farmers.

As shown in Table 3, it reflects the authenticity of lending behavior among farmers. The proportion of borrowing behaviors that occurred 2.3 times was the highest, accounting for $60.2 \%$, and the proportion of borrowing behaviors that occurred once was the largest, indicating that private lending behaviors are more common. Such frequent borrowing reflects the low level of farmers' income (consistent with the statistical 


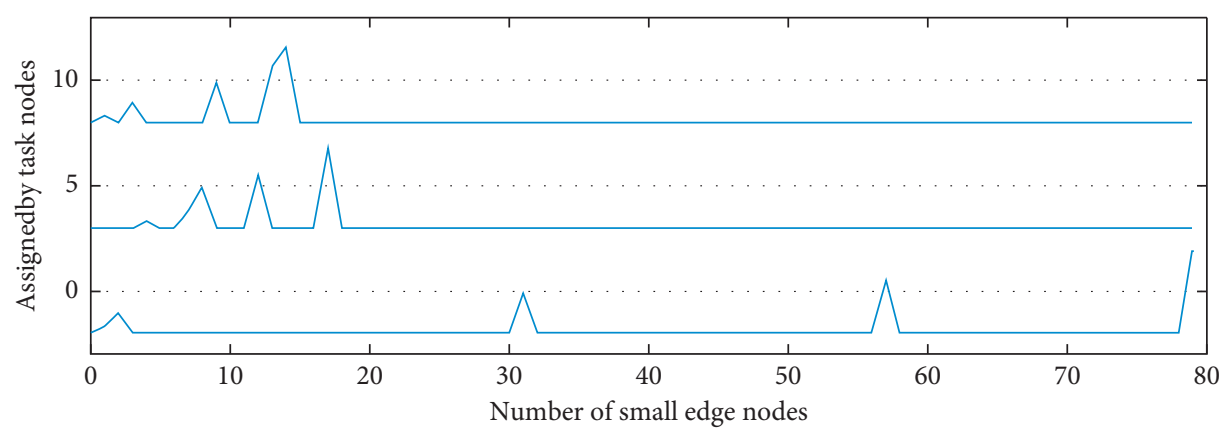

FIgURE 1: Node assignment task results.

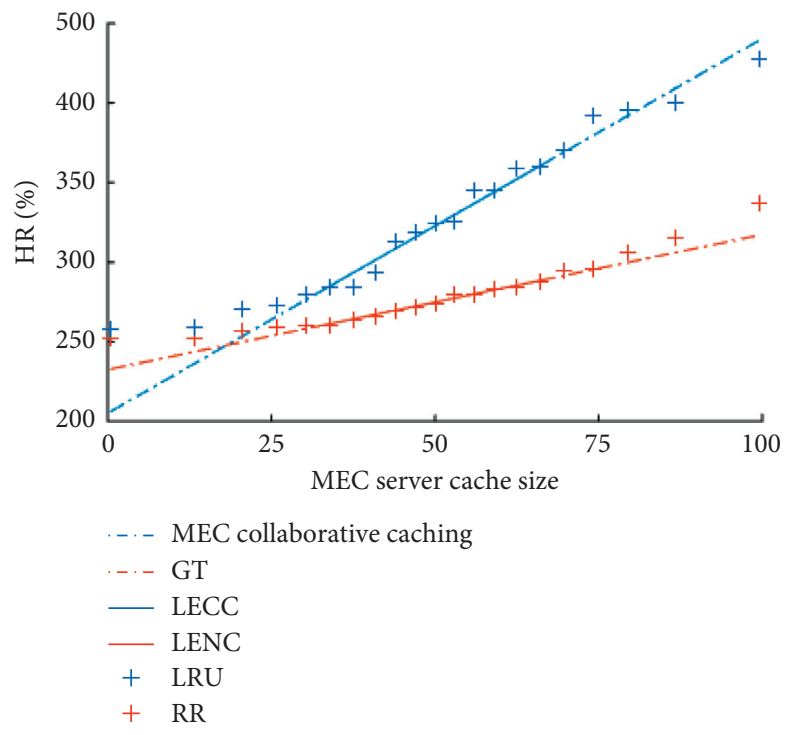

Figure 2: Performance comparison of cache mechanisms under changes in the cache space.

TABLE 3: The frequencies of mutual fund behavior between peasants.

\begin{tabular}{lccccc}
\hline & & Once & $2-3$ & $3-5$ & $\begin{array}{c}5 \text { or } \\
\text { more }\end{array}$ \\
\hline Borrow & $\begin{array}{c}\text { Number of farmers } \\
\text { (people) } \\
\text { Proportion (\%) }\end{array}$ & 155 & 186 & 127 & 99 \\
\hline \multirow{3}{*}{ Loan out } & $\begin{array}{c}\text { Number of farmers } \\
\text { (people) }\end{array}$ & 194 & 60.2 & 40.7 & 31.7 \\
\hline & Proportion (\%) & 62.3 & 56.2 & 32.8 & 24.4 \\
\hline
\end{tabular}

results of farmers' annual net income), and it is difficult to meet the funding needs in a timely manner. The distribution of farmers' loan uses is shown in Figure 3.

As shown in Figure 3, it shows that farmers' loans are mainly used to improve their lives and expand reproduction. The aspects of life are mainly used to build houses, weddings and funerals, children's schooling, and hospitalization. The production aspects include purchasing seeds and fertilizers and expanding reproduction. The table reflects the diversification of the use of farmers' borrowings. According to statistical data, living borrowings are higher than productive borrowings, accounting for $66.5 \%$ and $33.5 \%$, respectively, indicating that farmers borrow money first to solve the problem of life and secondly to expand the problem of reproduction.

4.4. Risk Control Analysis. In the case of guaranteeing the normal operation of mutual funds, the user fees are appropriately collected, and different interest rates are set according to different periods as shown in Table 4 .

As shown in Table 4, members manage their own funds. The level of interest rates will not pose a threat to formal financial institutions. Through interest rate adjustments, members can be encouraged to apply for short-term loans, which not only embodies the spirit of mutual assistance but also recovers funds in a timely manner. The setting of differential interest rate is determined by the characteristics of mutual funds. For members who need long-term loans, members are encouraged to apply for long-term loans from regular financial institutions in ways that conform to market interest rates to increase liquidity and promote sustainable development. The risk control is shown in Figure 4.

As shown in Figure 4, the risk level of the project is "lower risk," and the project can be considered. Among the 


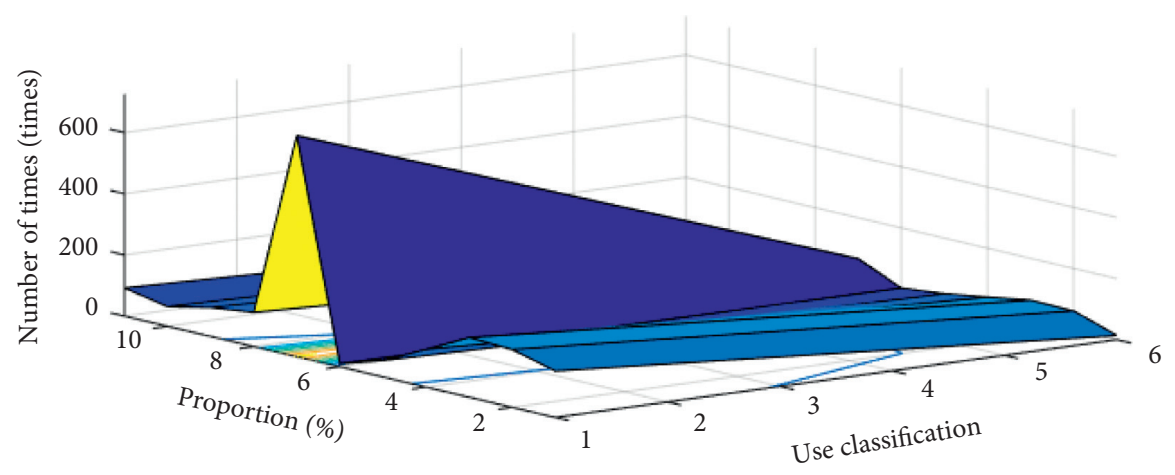

FIGURE 3: The specific distribution of mutual fund usage.

TABLE 4: Loan interest tables.

\begin{tabular}{lc}
\hline Loan term & Interest rate \\
\hline 10-day temporary loan & Interest-free \\
Within 6 months & Slightly lower than the RCC rate \\
$6-12$ months & Slightly higher than the rural credit cooperative rate \\
12 months and above & In line with market rates \\
\hline
\end{tabular}

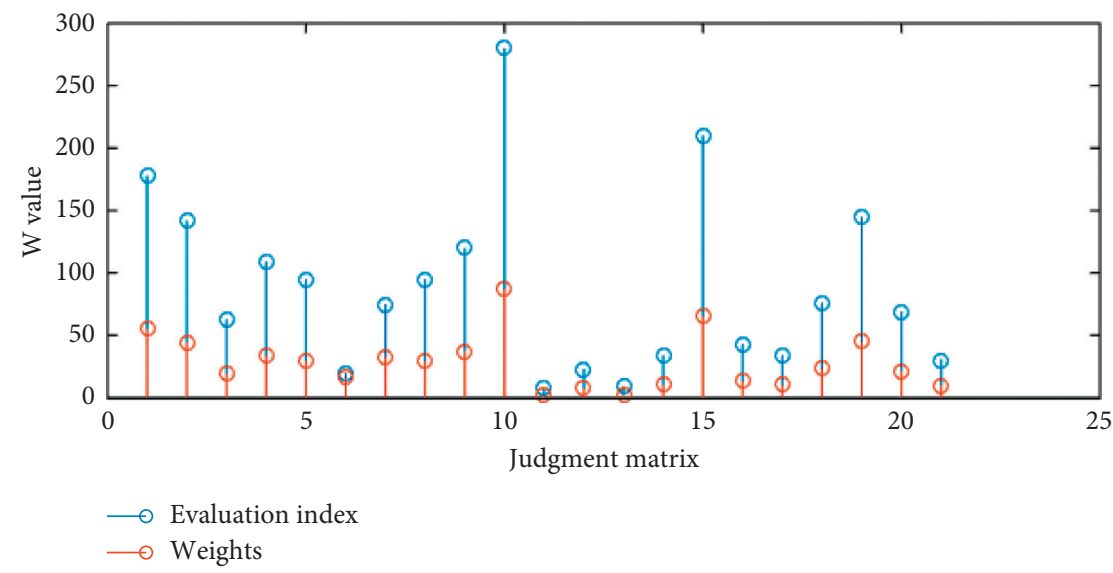

Figure 4: Weight of the judgment matrix and evaluation index.

project risk evaluation index weights, the energy-saving technology risk weight is 0.1415 and the market competition risk weight is 0.1848 , which are higher than other index values, and market competition has a strong advantage. As this project is a government-supported rural cooperative financial project, it has received preferential policies from the government in terms of funds, exchange rates, interest rates, and taxes. It basically does not consider the pressure brought by financing, but the risks generated by the project and its implementation. The obstacles encountered are the main considerations. Therefore, although the sources of funds for the new rural cooperative financial organizations are diverse, not every channel has played a role, so improving the ability to obtain funds is still achieved by finding the most effective way to make good use of existing channels. It is unrealistic to want to rely on meager loan interest for long-term development. The most practical way is to increase the income level of the organization itself.

\section{Conclusions}

With the rapid development of mobile multimedia services such as high-definition video streaming and virtual reality, mobile communications will play an increasingly important role in people's production and life. At the same time, smart terminals such as smart phones and tablet computers are rapidly spreading, and the data traffic generated by mobile terminal devices has exploded. In the future $5 \mathrm{G}$ mobile communications, mobile internet applications will continue to develop rapidly, the types of data services will be more diversified, the data traffic in mobile networks will continue to grow, and the next generation of mobile communication systems will face huge challenges. For these reasons, this article aims at the practical application of MEC in the risk control of new rural cooperative financial organizations.

In terms of the practical application, the new rural cooperative financial organizations have not established a sound risk control mechanism. The method of this article 
can improve the loan fund management mechanism of mutual funds, which is conducive to standardized management and risk control and guides the sustainable development of mutual aid organizations. Optimize the organizational structure of mutual fund cooperation organizations, adhere to the membership system, democratic management, and autonomous mutual assistance, and ensure the true nature of the mutual fund cooperation system. There are still some shortcomings in the research of this paper, for example, because the learning effect of the transfer learning method is directly affected by the degree of correlation between the source and target domains, when the degree of correlation is low, even the phenomenon of negative transfer may occur, and due to the multimedia content, the characteristics of diversity and fast update make the collection of samples with high similarity with the target domain in the content cache of the 5G-oriented edge network more complicated and difficult. Therefore, in the future, we need to further solve the negative migration of TL problems or explore more efficient big data technologies to improve the prediction accuracy of content popularity, thereby further improving cache performance.

\section{Data Availability}

No data were used to support this study.

\section{Conflicts of Interest}

The authors declare that they have no conflicts of interest.

\section{Acknowledgments}

The authors thank the financial support of the Start-Up Fund for Doctoral Research in Nanchang Institute of Science and Technology (NGRCZX-21-01).

\section{References}

[1] S. Jeong, O. Simeone, and J. Kang, "MEC via a UAV-mounted cloudlet: optimization of bit allocation and path planning [J]," IEEE Transactions on Vehicular Technology, vol. 67, no. 3, pp. 2049-2063, 2016.

[2] Y. Mao, J. Zhang, S. H. Song et al., "Stochastic joint radio and computational resource management for multi-user mobileedge computing systems," IEEE Transactions on Wireless Communications, vol. 16, no. 9, pp. 5994-6009, 2017.

[3] K. Zhang, Y. Mao, S. Leng et al., "Mobile-edge computing for vehicular networks: a promising network paradigm with predictive off-loading," IEEE Vehicular Technology Magazine, vol. 12, no. 2, pp. 36-44, 2017.

[4] P. Corcoran and S. K. Datta, "Mobile-edge computing and the Internet of things for consumers: extending cloud computing and services to the edge of the network," IEEE Consumer Electronics Magazine, vol. 5, no. 4, pp. 73-74, 2016.

[5] K. Zhang, S. Leng, Y. He et al., "Mobile edge computing and networking for green and low-latency Internet of things," IEEE Communications Magazine, vol. 56, no. 5, pp. 39-45, 2018.

[6] P. Bellavista, S. Chessa, L. Foschini, L. Gioia, and M. Girolami, "Human-enabled edge computing: exploiting the crowd as a dynamic extension of mobile edge computing," IEEE Communications Magazine, vol. 56, no. 1, pp. 145-155, 2018.

[7] L. Tang and S. He, "Multi-user computation offloading in mobile edge computing: a behavioral perspective," IEEE Network, vol. 32, no. 1, pp. 48-53, 2018.

[8] Y. Shen, K. Obermayer, and W. Stannat, "On average risksensitive markov control processes," Mathematics, vol. 51, no. 51, pp. 3652-3672, 2015.

[9] I. Ge, A. Rudolph, N. Shivappa et al., "Dietary inflammation potential and postmenopausal breast cancer risk in a German case-control study," The Breast, vol. 24, no. 4, pp. 491-496, 2015.

[10] A. Ramlatchan, M. Yang, Q. Liu et al., "A survey of matrix completion methods for recommendation systems," Big Data Mining and Analytics, vol. 1, no. 4, pp. 308-323, 2018.

[11] C. Zhang, M. Yang, J. Lv et al., "An improved hybrid collaborative filtering algorithm based on tags and time factor," Big Data Mining and Analytics, vol. 1, no. 2, pp. 128-136, 2018.

[12] Y. Liu, S. Wang, M. S. Khan et al., "A novel deep hybrid recommender system based on auto-encoder with neural collaborative filtering," Big Data Mining and Analytics, vol. 1, no. 3, pp. 211-221, 2018.

[13] H. Liu, H. Kou, C. Yan et al., "Link prediction in paper citation network to construct paper correlation graph," EURASIP Journal on Wireless Communications and Networking, vol. 2019, no. 1, 2019.

[14] W. Gong, L. Qi, and Y. Xu, "Privacy-aware multidimensional mobile service quality prediction and recommendation in distributed fog environment," Wireless Communications and Mobile Computing, vol. 2018, p. 8, Article ID 3075849, 2018.

[15] X. Zhang, W. Dou, Q. He et al., "LSHiForest: a generic framework for fast tree isolation based ensemble anomaly analysis," in Proceedings of the 33rd IEEE International Conference on Data Engineering (ICDE2017), pp. 983-994, San Diego, CA, USA, April 2017.

[16] Y. Xu, L. Qi, W. Dou et al., "Privacy-preserving and scalable service recommendation based on SimHash in A distributed cloud environment," Complexity, vol. 2017, p. 9, Article ID 3437854, 2017.

[17] X. Chi, C. Yan, H. Wang et al., "Amplified locality-sensitive hashing-based recommender systems with privacy protection," Concurrency and Computation: Practice and Experience, 2020.

[18] X. Xu, X. Zhang, H. Gao et al., "BeCome: blockchain-enabled computation offloading for IoT in mobile edge computing," IEEE Transactions on Industrial Informatics, vol. 16, no. 6, pp. 4187-4195, 2020.

[19] X. Xu, X. Zhang, M. Khan et al., "A balanced virtual machine scheduling method for energy-performance trade-offs in cyber-physical cloud systems," Future Generation Computer Systems, vol. 105, pp. 789-799, 2020.

[20] X. Xu, R. Mo, F. Dai et al., "Dynamic resource provisioning with fault tolerance for data-intensive meteorological workflows in cloud," IEEE Transactions on Industrial Informatics, vol. 16, no. 9, pp. 6172-6181, 2020. 